\title{
An algorithm to "clean" close stellar companions
}

\author{
H. Markov ${ }^{1}$, T. Valtchev ${ }^{1}$, J. Borissova ${ }^{1}$, and V. Golev ${ }^{2}$ \\ 1 Institute of Astronomy, Bulgarian Academy of Sciences, 72 Tsarigradsko chaussèe, BG-1784 Sofia, Bulgaria \\ 2 Astron. Obs. of University of Sofia, P.O. box 36, BG-1504 Sofia, Bulgaria
}

Received February 23; accepted June 15, 1996

\begin{abstract}
In our effort to study the post-main-sequence evolution of the globular cluster stars we developed some procedures which were designed to perform stellar photometry in moderately crowded fields by using a personal computer. This paper presents a description of an algorithm for cleaning close stellar companions which is included in our data processing. The main goal is to establish how the cleaning procedure biases the accuracy of the derived photometry. As a test for our data reduction, $B V$ photographic photometry for nearly 500 stars, down to 0.6 arcmin from the center of globular cluster M $5=$ NGC $5904=$ C $1516+022$ has been performed. In addition, we present a comparison of our photometric results with those obtained with the DAOPHOT package for the same cluster region. We also discuss the ability of three kinds of magnitudes - based on Gaussian fits, the use of synthetic apertures and the output of the DAOPHOT package - to reveal morphological features in the colormagnitude diagram.
\end{abstract}

Key words: globular clusters: individual: M 5 technique: image processing

\section{Introduction}

Research of the post-main-sequence evolution of the globular cluster (GC) stars raises the necessity of stellar photometry in the most inner parts of the clusters. Penetrating into the cluster centers the astronomers aim to improve, statistically, the sample of bright stars occupying the horizontal branch (HB) and the red giant branch (RGB) with its asymptotic features (AGB) and thus to express more reliably some morphological peculiarities of these branches predicted by the existing theories.

Stellar photometry in crowded fields is a fairly sophisticated task and in the last two decades many powerful packages which help astronomers to carry out such kind of photometry have been created - ROMAFOT (Buonanno

Send offprint requests to: $\mathrm{H}$. Markov et al. 1983; Buonanno \& Iannicola 1989), DAOPHOT (Stetson 1987) etc. All of them, however, are designed to work under operating systems serving VAX machines or workstations and only few programs exist under DOS for PCs. Having no regular access to such packages we were forced to develope our own computerized procedure for doing stellar photometry in moderately crowded fields using a PC. The basic ideas of such procedures are widely discussed in the literature (Buonanno et al. 1979; Newell 1979; Stetson 1979 etc.). An important step in our reduction procedure is cleaning close stellar companions, a procedure which can be applied in aperture photometry or other kinds of photometry based on modelling stellar images (like a Gaussian fit) of the inner parts of globular clusters. As a target for testing our image processing tools we choose the GC M5 which is an example of a well investigated cluster (Arp 1962; Simoda \& Tanikava 1970; Buonanno et al. 1981; Brocato et al. 1995 etc.) and for which we have a good $U B V$ set of short exposure plates. The data processing had been already completed when we got a possibility to use DAOPHOT (version before 1989) - a package especially designed for stellar photometry in crowded fields. A comparison of the results obtained by two different methods, ours and DAOPHOT's, seemed to us very useful as it would allow us to check whether the achieved accuracy depends on the reductions applied or whether the intrinsic accuracy limits of observing technique (photographic emulsion in our case) and the manner of digitization have been reached. The results of this comparison are presented below. Section 2 deals with the observations, digitization, the manner the star images were located, emulsion linearisation and some data filtering procedures we have used. Section 3 describes the algorithm we have developed to clean stellar companions and derive instrumental magnitudes. In Sect. 4 we comment on our derived results, and conclusions about the application of the cleaning procedure are drawn. 


\section{The observational material and preliminary procedures}

The observational material which supports this investigation consists of four plates of M $5=$ NGC $5904=$ C $1516+022$ - two in $B$ and two in $V$ - taken at the $\mathrm{RC}$ focus of the National Astronomical Observatory $2 \mathrm{~m}$ telescope $(F / 8$, scale 12.86 arcsec per $\mathrm{mm})$. The $B$ band is realized with ORWO ZU21 behind GG13 emulsion-filter combination, and the $V$ band - via KODAK 103a-D behind GG11. All plates are taken with very short exposure times (40 s. in $V$ and $90 \mathrm{~s}$. in $B$ ). This allows good separation of the stars down to 0.6 arcmin from the cluster center.

\subsection{Digitisation and subframes' design}

On each plate the cluster image out to about 6 arcmin of the center was divided into nine overlapping square regions (18 $\mathrm{mm}$ in size) which were digitized with the JoyceLoeble microdensitometer of the NAO. For the simplicity of the future explanation the digitized cluster regions we call these "frames". It was rather time consuming to scan such a large area (more then $2500 \mathrm{~mm}^{2}$ ) but our hope was to indentify as many stars already photometered by other investigators as possible in order to be used as a reference system. The plates were traced with a $50 \mu \mathrm{m}$ square diaphragm and a $30 \mu \mathrm{m}$ step in both $X$ and $Y$ directions in agreement with the fact that the size of the smallest measured image on the plates was evaluated to be about 2 arcsec. Having no appropriate finding routines, for the sake of star location, we accepted an approach close to that described in Stetson (1979) and Chiu et al. (1979), namely to process the subframes centered on the star under consideration. The manner in which subframes were designed is presented below. Every four frames corresponding to one and the same cluster region on the different plates were adjusted to make the positions of the stars coincide with each other. Then displaying one of the plates (frame by frame) smaller arrays (subframes) relevant to individual star images were formed by extraction. During this procedure we prepared a list where all stars in contact were registered. By the help of some PCVISTA routines arranged in a batch mode, the process of subframes formation was automatically performed on the other three plates (due to their position adjustment). In this way about 1000 subframes $(40 \times 40 \mu \mathrm{m})$ each centered on an individual star were obtained from every plate. Our experience showed that the scatter of the star's centers (according to different plates) in both directions was less than 2 pixels. There are many reasons for such a scatter: the orientation of the plates on the scanning desk, the frames adjusting procedure etc. For the purposes of the present study the achieved accuracy of the star's centering was regarded as good.

\subsection{Linearisation of the emulsion}

As we have mentioned in the introduction our photometric procedure yields two sets of MI as an output: one based on a Gaussian fit and the other - on a synthetic aperture. In the former case we used data in photographic densities since in the fitting routine a term considering the local sky background variations had been included. But in the second one for the sake of a correct background evaluation we had to work in relative intensities. The input data for DAOPHOT (the output magnitudes of which we used to check the above two sets) also have to be in relative intensities. On the used plates there was not any additionally exposed photometric pattern - wedge or spots. To transform photographic densities to relative intensities we applied an internal calibration method using density profiles of stars with known magnitudes. For this purpose 24 of Arp's electrophotometric standard stars, previously recognized in the cluster region under consideration, were used. These stars were scanned additionally, each in a single frame $(40 \times 40$ pixels $)$, under the same conditions as described in Sect. 2.1. A comprehensive description of the procedure is given in Markov (1994).

\subsection{Filters used}

There were two independent reasons for which we find it necessary to use a certain data filtering. First, an inspection to the stellar images revealed that the frames had transposed rows probably due to the inertness of the microdensitometer, and manner of scanning - in order to save time, every scanned row was made in the opposite direction to the previous one. Unfortunately, the observed displacement is not an exact number of pixels - it is less than a pixel. To improve the situation we need some kind of filtering like a running average with normalized weights. The second reason is connected with the reliable determination of the stellar centroid in the crowded central field. Our presumption was: rejecting the high frequencies to symmetrize the PSF, to improve the determination of the PSF and to get more reliable star locations as well. In order to solve both problems using one reduction procedure we found it appropriate to apply the discrete wavelet transformation (DWT) but in different stages of its application. The wavelet transformation is a versatile tool for signal and image processing and it is used very widely. Thanks to this there are many papers which describe the theory, numerical algorithms and applications of the wavelet transformation (Starck 1993; Starck \& Murtagh 1994 etc.). In our work we applied the so called trous algorithm of the DWT described in Starck (1993). The physical basis of this transformation is grounded on consecutive filtering of the frames with a low-pass filter a running average. The difference between two consecutive resolutions yields the discrete wavelet transformation at a given level. To restore frames with transposed rows 
we used the first smoothing level of the DWT. The resolution losses are expected to be negligible, since on the best plate the PSF has a FWHM of about 5 pixels but the smoothing window chosen is about 3 pixels in diameter. Figure 1 presents one enlarged rough stellar image (left) and its smoothed version (right). All scanned regions were treated in this manner.

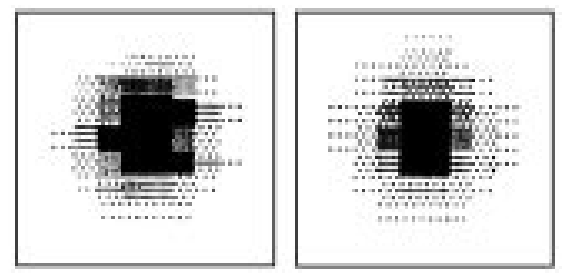

Fig. 1. Restoration of an image distorted by the transposition of rows. The left panel shows one scanned stellar image - the rows' displacement is probably due to the inertness of the scanning machine. The right panel demonstrates the same image but treated with the first smoothing level of the wavelet transformation

For solving the second problem we used the zero DWT level - namely its ability for expressing image features with a desired size (for example - stellar peaks) more clearly. The effect of its application is demonstrated in Fig. 2, right panel, while the left presents the original frame. The improved separation of the stellar images on the right panel which are in a contact on the left is more than evident. The wavelet transformed frames were chosen only as an input for DAOPHOT's star-finding algorithm.

\section{Description of the cleaning algorithm and the accuracy test}

The entire data processing was done by using a PC 486/DX2. The software, written on PASCAL, was designed to process subframes centered on the stellar images and consists of two basic procedures - cleaning of close companions and instrumental magnitudes derivation.

\subsection{Cleaning algorithm}

To suppress stellar companions Newell (1979) has proposed a symmetry-clean procedure, which operates by comparing the intensities of all pairs of pixels diametrically opposite with respect to the image center. The author included this procedure into the centering procedure aiming to increase the speed and reliability in crowded field centering. Supposing a circular symmetry of the stellar image and taking into account the variation of the grain noise over its profile (Lee \& van Altena 1983) we have developed another mechanism to clean close companions. First, the image was divided into a few contiguous annular regions with a width of about one pixel. The last few annuli should be sufficiently far from the center of the star - several times the FWHM of the image - so that its light contribution to the background determination is negligible. If the image is not damaged by close companions the density distribution within each annulus will be symmetric with coinciding values of the mode, median and mean. In the opposite case defining the mode as the most frequently occurring density value in a given annular region we stated the following principle of cleaning: any pixel value exceeding the mode with some predetermined multiple of the sample standard deviation, $k * \sigma$ (where $k$ is a real number), was considered as "improper" for the annular distribution under consideration and replaced with the mode value. The pixel density histogram of the regions in question is usually not actually calculated, instead the mode of the distribution is estimated from the formula (e.g. Kendall \& Stuart 1977, p. 40):

mode $=3 *$ median $-2 *$ mean

provided that the median is less than the mean. Otherwise, the mean can be taken. A comprehensive discussion of using the mode as the most representative value of a sample of density values one can find in Stetson (1987) and Da Costa (1992). Practically the scheme works as follows: first, to determine the median and mean any strongly deviant pixel density within each annulus is clipped iteratively until $\sigma$ stabilizes; second, the mode of the distribution is calculated and than the outlined cleaning procedure is applied. The procedure is fully automated and does not need any investigator's interaction. Here we have to emphasize that the efficiency of the cleaning procedure depends on many factors and mainly on the number of companions and their closeness. Figures 3a and 4a illustrate the effect of applying the above described procedure in the two dimensional plane for two different cases. The frames are centered on the stars under consideration. In both cases, the original image is presented on the left but its cleaned version on the right panel. In addition, we present in Figs. 3b and $4 \mathrm{~b}$ profiles of the same stars. These are not a simple image cross-section in a given direction but rather integrated profiles which include all points found within the image - it is a plot of the densities found on a given radius. The presentation of the original and cleaned profiles are the same as for the density maps.

\subsection{Instrumental magnitudes and accuracy test}

The image cleaned in this manner was used for magnitude index formation both through a multi-component bi-dimensional Gaussian fit (using data in photographic densities) and with a synthetic square aperture with constant size (in relative intensities). The size of the aperture is 4 pixels in each dimension. The application of a oneor bi-dimensional Gaussian fit (GF) to determine $\mathrm{MI}$ as well as the application of synthetic aperture (SyA) photometry are well documented in the literature (Stetson 

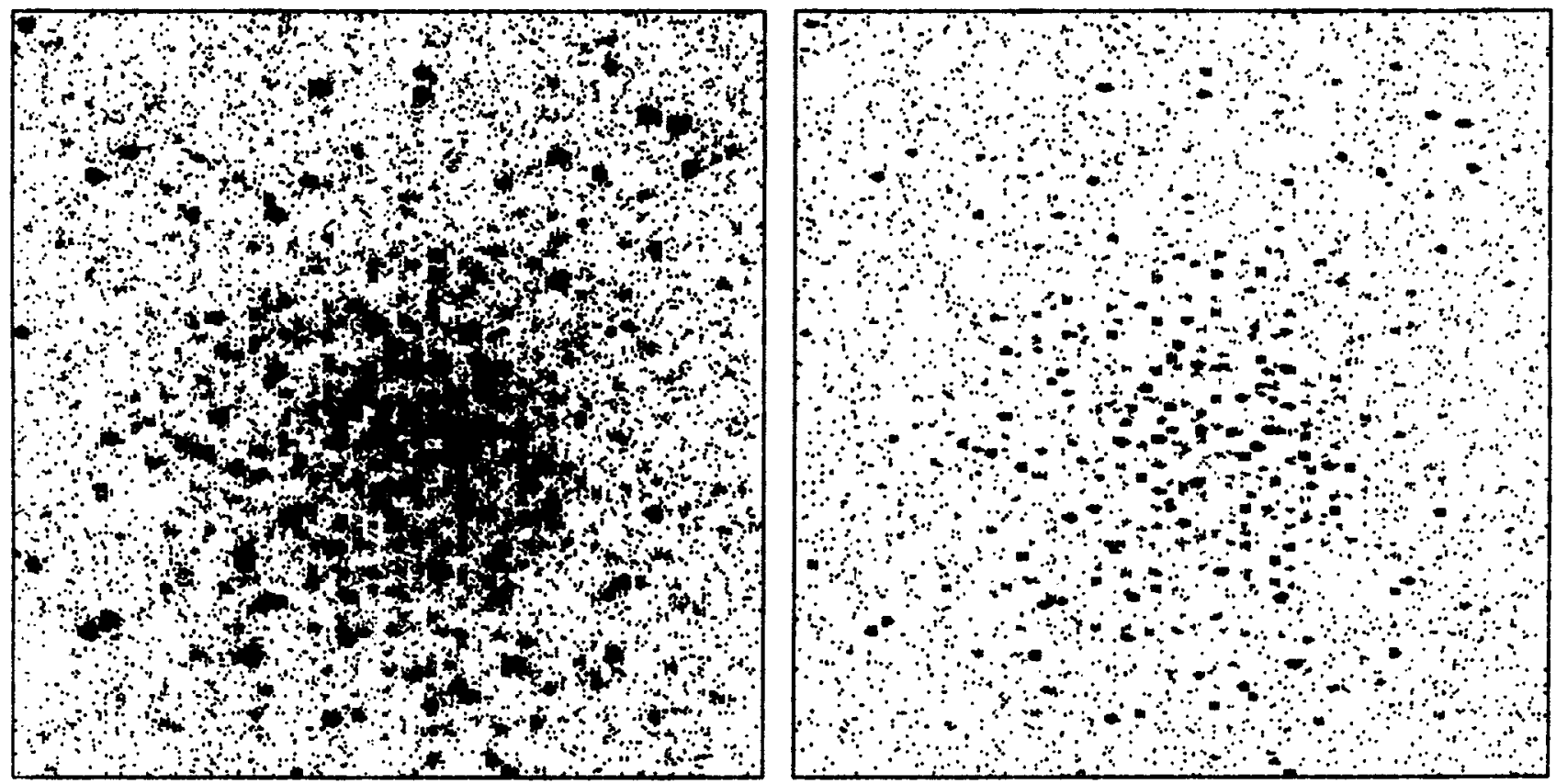

Fig. 2. Two frames of one and the same cluster region. On the right hand side the zero DWT level of the original frame (left panel) is shown. The gain in resolution is well demonstrated. Improving the separation of the contact images we aim to get more reliable stellar centroids
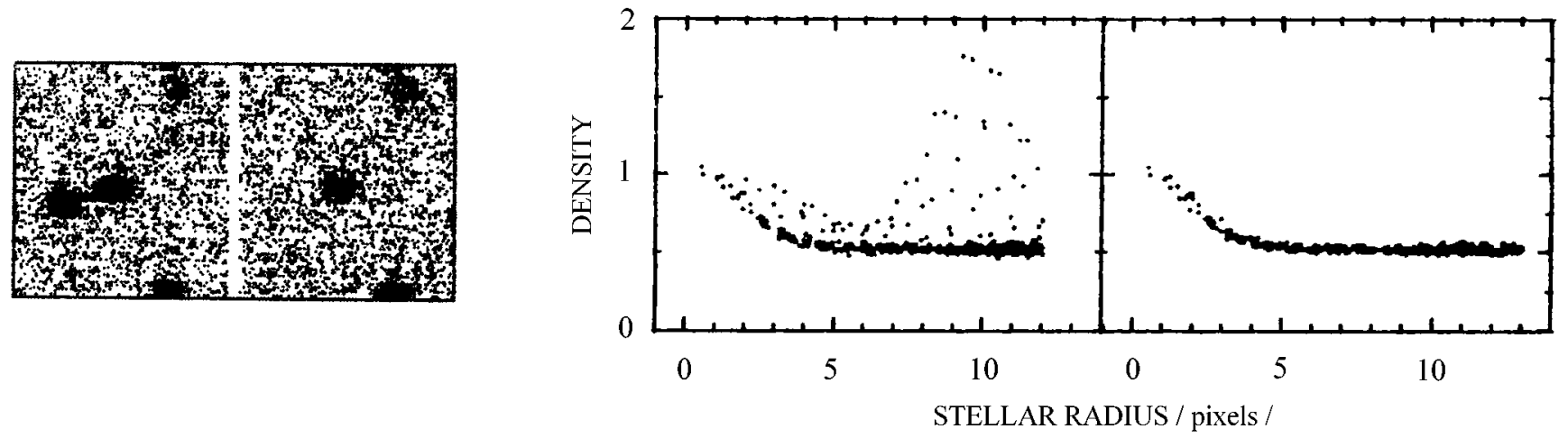

Fig. 3. a and b) Illustration of the efficiency of the cleaning algorithm: a) two dimensional density maps of the stellar images centered on the star under consideration (the left panel shows the original but the right - the already cleaned image); b) integrated stellar profile of this star - original (left) and cleaned (right)
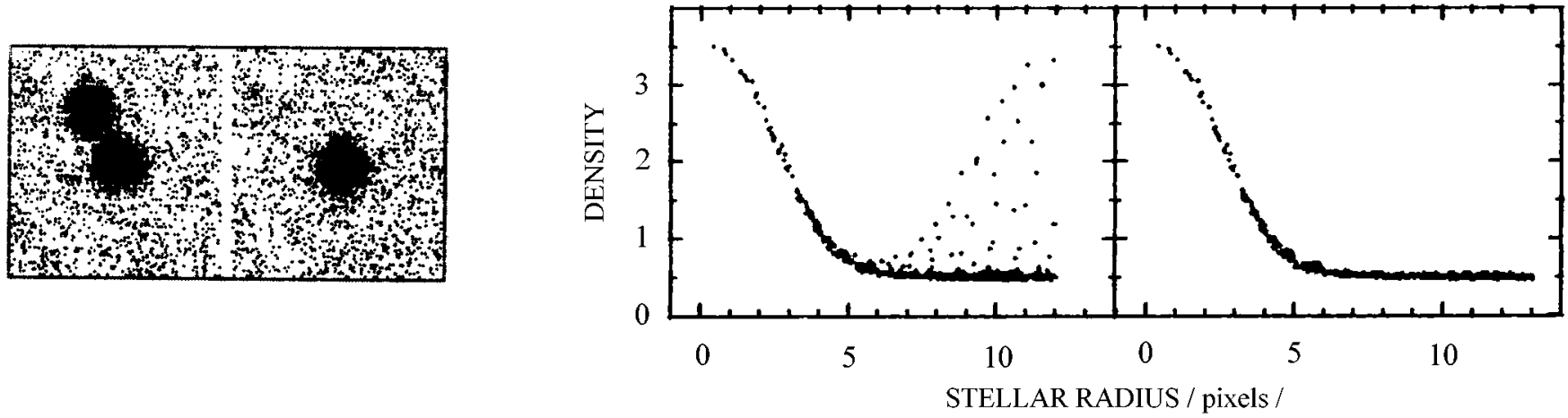

Fig. 4. a and b) The same as Fig. 3 but for another image 


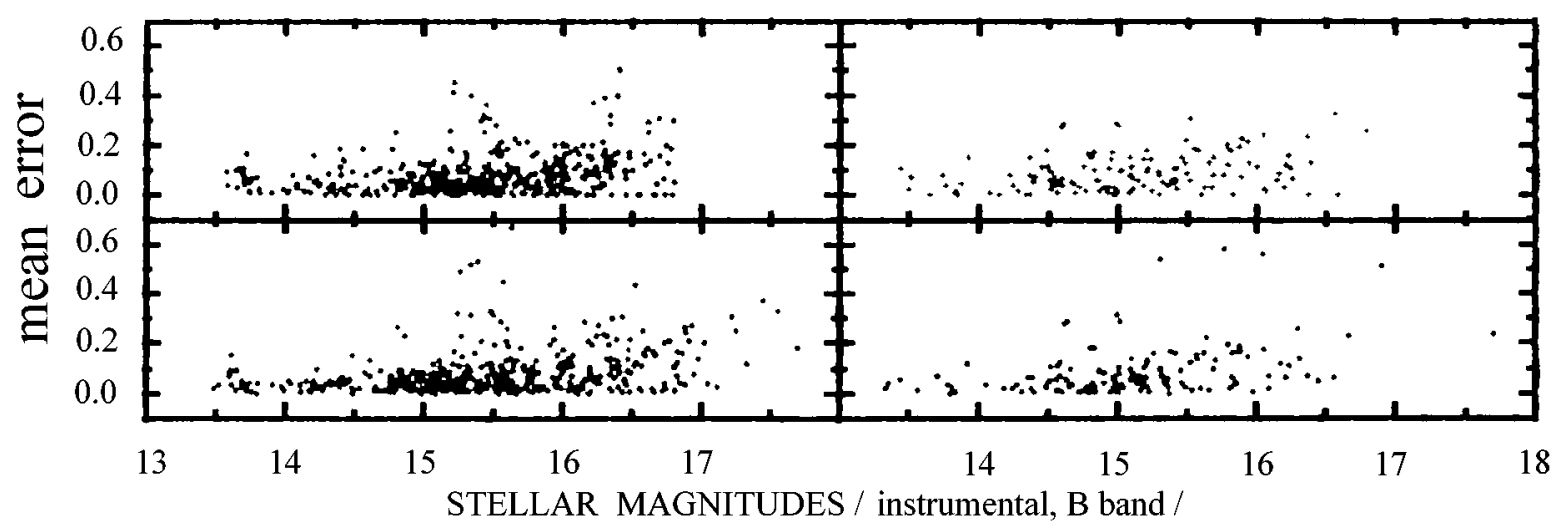

Fig. 5. Mean error of the instrumental magnitudes for the two plates in the $B$ band as defined in Sect. 3.2. The lower panels present the comparison of instrumental magnitudes determined with a synthetic apperture with constant size for isolated stars (left) and stars with close companions (right). The upper panels - the same as the lower but for magnitudes derived with bi-dimensional Gaussian fits of the stellar images. The observed trend of the errors on the right panels is the same as on the left. This fact shows that the applied cleaning procedure does not bias the cleaned image in any way

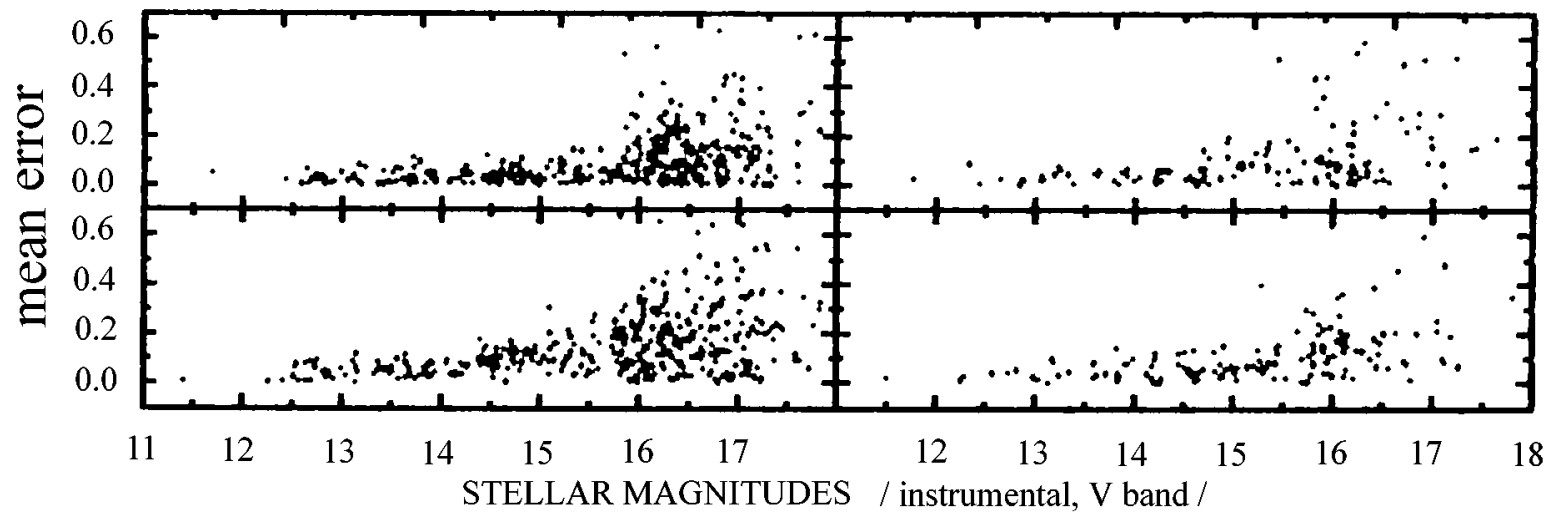

Fig. 6. The same as Fig. 5 but for $V$ magnitudes

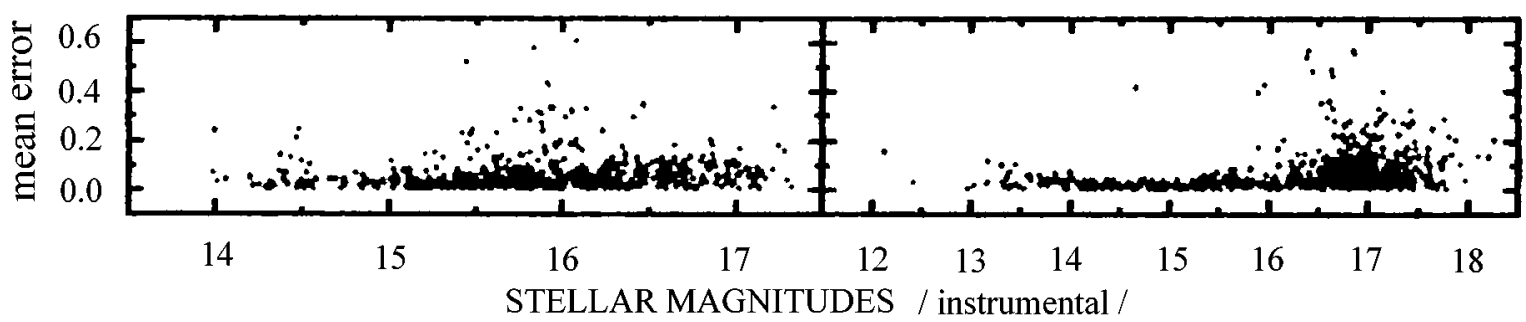

Fig. 7. Mean error of the instrumental magnitudes as an ouput from DAOPHOT for two plates in $B$ (left) and $V$ (right)

1979; Buonanno et al. 1979; Stetson 1990 etc.) and will not be discussed here. DAOPHOT photometry was performed in a standard way and the derived magnitudes will be refereed as "PSF magnitudes" hereafter. To complete an unique system of instrumental magnitudes, the MIs of the same colour were averaged together and then the individual plates were transformed to the average plate using a least-squares polynomial. These fits permit the mean er- ror of a MI on a typical plate to be estimated from the transformation residuals (Stetson 1979):

$\hat{\sigma}^{2}\left(m_{i}\right)=\frac{1}{n-1} \sum_{n}\left(m_{i, \text { transformed }}-m_{i, \text { averaged }}\right)^{2}$

where $n$ is the number of plates used but $m_{i}$ is the average of the transformed magnitude of the $i$-th star measured on $n$ plates. Figures 5 and 6 demonstrate the mean 
error in the $B$ and $V$ bands for a Gaussian fit (upper panels) and SyA magnitudes (lower panels), respectively. The left panels show the mean error for isolated stars, and the right pannels - the same for stars in contact. We prefer to demonstrate both kind of instrumental magnitudes because their determination differs. In Fig. 7 are presented the mean error in the $B$ (left) and $V$ (right) bands for PSF-magnitudes (DAOPHOT output).

\section{Discussion and conclusions}

Comparing left and right panels of Figs. 5 and 6, one may expect if the cleaning procedure disturbs in some way statistical properties of the image, the mean error to be more significant or the demonstrated dependence to have a different shape for stars in contact - that is not observed in the figures. This is an important result which demonstrates that the applied cleaning procedure does not bias the photometry in any way. Thanks to this we were able to penetrate the cluster center down to 0.6 arcmin even using aperture formed MIs - which usually are not recomended for crowded fields. The problem here is not the size of the used aperture - we can chose it sufficiently small to avoid close companions - but rather the statistical properties of the pixels used for the background evaluation. In our case, the area used for the background evaluation, being previously treated with the cleaning procedure, is free from highly deviant values. One third of the stars measured, were classified as stars in contact and most of them have neighbours which would prevent a correct background determination, if not rejected. We want to comment here another field where the cleaning algorithm can be successfully applied. Namely for example in the DAOPHOT package it is known that to determine the PSF for a given frame some well isolated stars should be chosen. In crowded fields such stars are very often difficult to find or even can not be found at all in the frame being processed. In such a case, the cleaning procedure can be used to free the environment of the selected stars from the influence of companions as one preventive step in order to determine reliable PSF.

For the sake of completeness we like to comment on two results which were derived as by-products of our investigation. The first one is the worse internal accuracy of $B$ magnitudes in comparison with those of $V$ evident in Figs. 5 and 6 . This fact can be explained only with the used emulsion - ORWO ZU21 - which we had to use to fulfil the $B$ band. The scatter in $B$ badly influences the horizontal branch stars and will probably make it difficult to analyze the luminosity function.

The second result we want to mention here is the comparison of the accuracy derived by our data reduction procedure and by DAOPHOT. The relations presented in Figs. 5 and 6, on the one hand, and in Fig. 7, on the other, show that the accuracy of our photometry is, as a whole, about $20 \%$ poorer than that derived via DAOPHOT.

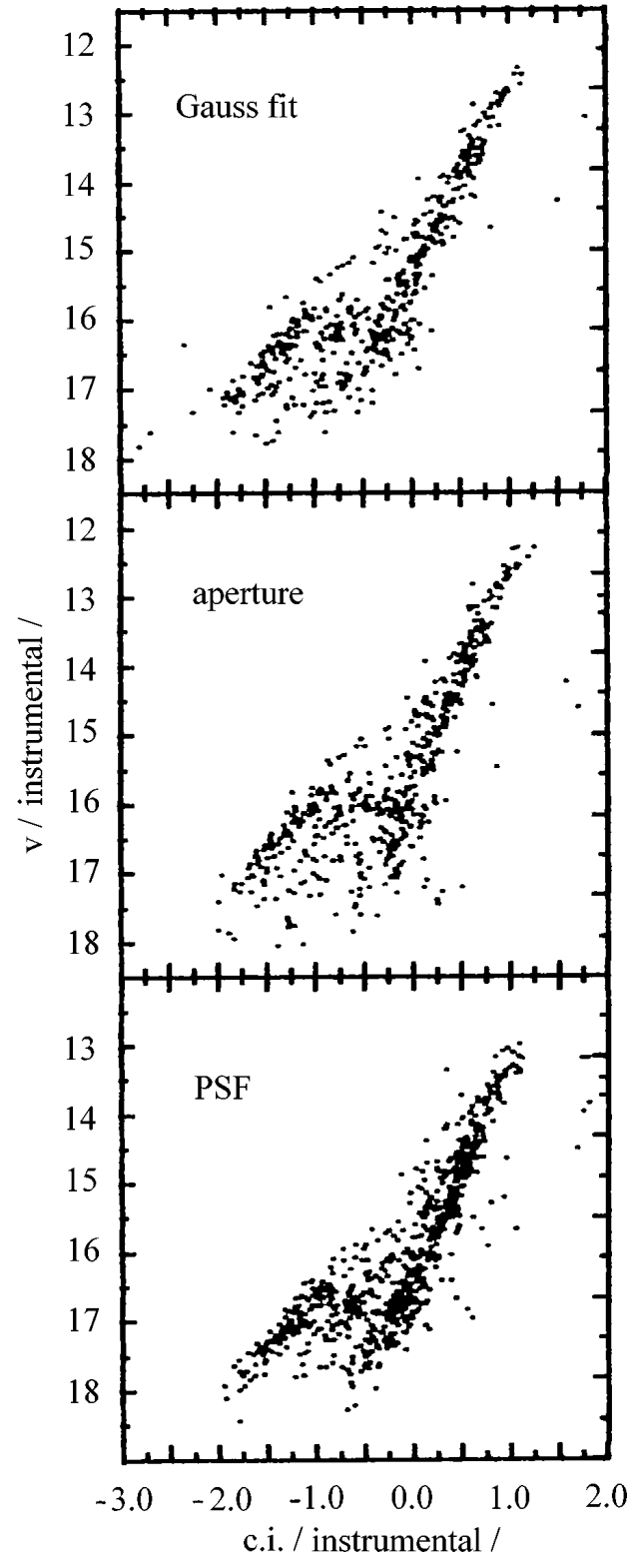

Fig. 8. Color-magnitude diagrams in the instrumental system for about 500 stars measured down to 0.6 arcmin from the M 5 cluster center. The diagrams are based on different sets of magnitudes: Gaussian fit (top), synthetic aperture (middle) and DAOPHOT output (bottom)

Obviously the achieved accuracy is mainly determined by the procedures used for deriving the magnitude index. To evaluate the practical meaning of this fact, as a final result we consider the color-magnitude diagrams by using different kinds of magnitudes. Figure 8 presents three CMDs based on Gaussian fit (top), synthetic aperture (middle) and the output of DAOPHOT package (lower) instrumental magnitudes - which we find representative enough for the aims of this investigation. The diagrams include measurements for about 500 stars placed in the central 


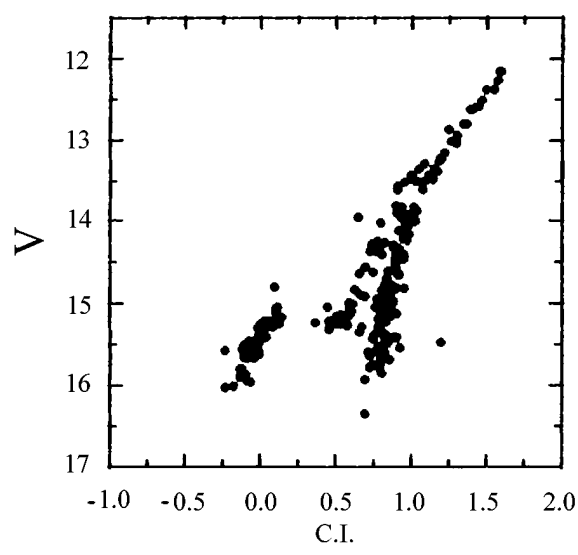

Fig. 9. Color-magnitude diagram of the GC M 5 (Buonanno et al. 1981)

region of M 5 down to 0.6 arcmin from the cluster center and are not cleaned for variables and field stars. For comparison in Fig. 9 we display the M 5 CMD derived by Buonano et al. (1981) for stars in the annulus with radius $2 \leq r \leq 5.6$ arcmin. It is evident from Fig. 8 that even in the instrumental system the main branches are well outlined by all three magnitude sets. In our opinion, for the faint stars DAOPHOT magnitudes are more reliable but those from a Gaussian fit are not dramatically different. Unfortunately measurements of horizontal branch are affected by the exposure duration of the plates which were aimed to resolve the cluster center. The completness test as well as the transformation to the reference system and the whole analysis of the color-magnitude diagram are subject of the next investigation where we intend to include the $\mathrm{U}$ plates too.

As a result of our investigation we find that the present cleaning algorithm can successfully be applied to help stellar photometry in moderately crowded fields, in cases of the absence of more powerful packages. Satisfactory results can be achieved even using simple methods for magnitude index formation - like a synthetic aperture for example.
Acknowledgements. The authors are grateful to Dr. Nedka Spasova (Astron. Inst., Sofia) who took part in data processing and kindly discussed with us the strategy of this investigation. Special thanks Dr. Tsvetan Georgiev (NAO) for advising us some helpful articles. This research is supported by Bulgarian National Scientific Foundation grant under contract No. F-328 with the Bulgarian Ministry of Science and Education.

\section{References}

Arp H.C., 1962, ApJ 135, 311

Auer I.H., van Altena W.F., 1978, AJ 83, 531

Brocat E., Castellani V., Ripepi V., 1995, AJ 109, 1670

Buonanno R., Cors C.E., De Biase G.A., Ferraro I., 1979, in: Image Processing in Astronomy, Sedmak G., Capaccioli M. and Allen E.J. (eds.) Trieste, Italy, p. 354

Buonanno R., Corsi C.E., Fusi Pecci F., 1981, MNRAS 196, 435

Buonanno R., Buscema G., Corsi C.E., Ferraro I., Iannicola G., 1983, A\&A 126, 278

Buonanno R., Iannicola G., 1989, PASP 101, 294

Chiu L.-T.G., van Altena W.F., Stetson P., 1979, in: Image Processing in Astronomy, Sedmak G., Capaccioli M. and Allen R.J. (eds.) Trieste, Italy, p. 240

Da Costa G.S., 1992, in: Astronomical CCD Observing and Reduction Techniques, Howell S.B. (ed.) ASP Conf. Ser.

Kendall M., Stuart K., 1977, The Advanced Theory of Statistics, Vol. 1, Distribution Theory, 4th edition. Charles Griffin and Co., London

Lee J.-F., van Altena W.F., 1983, AJ 88, 1683

Markov H., 1994, A\&A 291, 1027

Newell E.B., 1979, in "Image Processing in Astronomy", Sedmak G., Capaccioli N. and Allen R.J. (eds.)

Simoda M., Tanikawa K., 1970, PASJ 22, 143

Starck J.-L., 1993, in MIDAS Manual, Release 93NOV, Image Processing Group, ESO, Garching

Starck J.-L., Murtagh F., 1994 (in press)

Stetson P.B., 1979, AJ 84, 1056

Stetson P.B., 1987, PASP 99, 191

Stetson P.B., 1990, PASP 102, 932 